\title{
Effects of lesions in the amygdalo-hippocampo-septal system on food and water intake in the rat
}

WILLIAM J. PIZZI ANO STANLEY A. LORENS ${ }^{1}$

INSTITUTE FOR JUVENILE RESEARCH, CHICAGO, ILLINOIS

Bilateral damage to the stria terminalis, stria medullaris, and postcommissural formix resulted in a sustained significant increase (at least 79\%) in daily water consumption. Food intake was only transiently affected. The results suggest that amygdaloid, septal and hippocampal regulation of water intake is mediated via these pathways.

Although it is generally accepted that hunger and thirst are regulated primarily by hypothalamic "centers," recent evidence suggests that certain limbictelencephalic structures are also involved in the regulation of food and water intake. Lesions in the septal area (Harvey \& Hunt, 1965; Wolfe et al, 1966), hippocampus (Kimble \& Coover, 1966), amygdala (Grossman \& Grossman, 1963) and cingulate gyrus (Lubar \& Wolfe, 1964) all have been reported to increase daily food and water consumption.

The present study was designed to determine whether the stria medullaris, stria terminalis, and post commissural fornix, efferent systems of the septal area, amygdala and hippocampus to medial preoptic and anterior hypothalamic areas and/or the habenular complex, play a role in regulating food and water intake. Method

The Ss were 12 male albino rats (Holtzman SpragueDawley strain) 90 days old and weighing 300-350 gm at the start of the experiment. The Ss were housed individually in $11 \times 9 \times 9$ in. cages. Room temperature and relative humidity varied between $72-80^{\circ} \mathrm{F}$ and $24-$ $55 \%$, respectively. Food (Purina Breeder Chow) and tap water were avallable ad lib. Body weight and food and water consumption were recorded daily, 14 days preoperatively and 28 days postoperatively. Water intake was measured by weighing the water bottles and converting to $\mathrm{ml}$. Control data indicated that errors due to leakage and evaporation were less than $1 \%$. Food intake was determined by placing a known amount of food in each cage and subtracting leftover portions both in and under the cage. Crumbs which fell through the cage were collected upon sheets of newspaper.

Lesions were produced stereotaxically under ether anesthesia by passing DC ( $3 \mathrm{~mA}$ for 45 sec.) through a cathode in the brain and an anode in the anus. The cathod was a 26 ga stainless steel needle insulated with Insulex except at the cross section of its tip. Sham operated Ss were treated in the same manner as lesioned Ss except that the electrode was never lowered into their brains.
The Ss were sacrificed with an overdose of Nembutal. Their brains were removed, frozen, and sectioned at $50 \mu$. Every fifth section was saved and stained with cresyl violet. The locus and extent of lesions were microscopically determined.

\section{Results}

The lesioned Ss sustained bilateral transection of the stria terminalis, stria medullaris, and descending columns of the fornix at the rostral pole of the thalamus. The nuclei fimbrialis and triangularis septi, the bed nuclei of the anterior commissure and stria terminalis, the parataenial and periventricular thalamic nuclei, and the dorsal one-fourth of the medial preoptic area were severly damaged in all Ss. The paraventricular and supraoptic nuclei were completely spared. The lesions were confined to the region depicted in Figs. 17-25, 1.0-1.5 mm lateral and -1.4+0.5 vertical, of König \& Klippel (1963).

As seen in Table 1, the lesioned Ss drank $128 \%$ more water than controls during the first postoperative week and 79-86\% more water during the second through fourth postoperative weeks. The lesioned Ss, furthermore, showed a significant increase $(23 \%)$ in food intake but only during the second postoperative week.

Although the body weights of the two groups were never significantly different, the lesioned Ss did weigh $3-6 \%$ less than controls throughout the postoperative period. For the first five postoperative days all lesioned Ss showed the rage response common to septally lesioned rats. All Ss appeared healthy postoperatively, no gross motor or other deficits being observed.

Table 1. Means, standard deviations, $t$ scores, and significance levels (determined by a two-tailed test for mean difference) for daily food (in grams) and water (in ml.) consumption of sham operated $(n=6)$ and lesioned $(n=6)$ rats.

\begin{tabular}{|c|c|c|c|c|c|c|c|c|c|c|}
\hline & & Wate & & & & & Foo & & & \\
\hline Group & p Sho & & Les & ion & & Sha & & Les & on & \\
\hline Week & $M$ & $S D$ & $M$ & SD & $\dagger$ & $M$ & SD & $M$ & SD & $t$ \\
\hline 2 & 33.6 & 8.7 & 36.2 & 4.3 & 0.66 & 20.2 & 2.3 & 19.9 & 1.6 & 0.26 \\
\hline$\overline{1}$ & 38.8 & 11.7 & 43.5 & 8.8 & 0.80 & 19.0 & 2.3 & 19.0 & 1.9 & 0.01 \\
\hline $\begin{array}{l}\text { Posto } \\
\text { Week }\end{array}$ & & & & & & & & & & \\
\hline 1 & 44.9 & 10.2 & 102.2 & 13.1 & $8.47^{*}$ & 18.7 & 2.4 & 22.3 & 4.3 & 1.80 \\
\hline 2 & 44.4 & 12.3 & 82.6 & 13.0 & $5.22^{*}$ & 19.8 & 2.4 & 24.3 & 2.3 & $3.37^{* *}$ \\
\hline 3 & 46.2 & 14.0 & 82.5 & 24.4 & $3 \cdot 17^{* *}$ & 19.6 & 2.8 & 20.2 & 1.7 & 0.50 \\
\hline 4 & 45.2 & 13.2 & 82.9 & 14.7 & $4.66^{*}$ & 19.2 & 2.1 & 20.7 & 2.2 & 1.24 \\
\hline
\end{tabular}




\section{Discussion}

This study has shown that simultaneous bilateral damage to the stria terminalis, stria medullaris, and descending columns of the fornix produces a dramatic and sustained increase in daily water intake. The data support the hypothesis that amygdaloid, septal and hippocampal regulation of water consumption is mediated via these pathways.

Further support for this hypothesis derives from the magnitude of the increased drinking observed in this study. Wolfe et al (1966) reported a mean $48 \%$ increase in water intake during the first nine days following septal lesions. Grossman \& Grossman (1963) found a $54 \%$ increase for the first two weeks following amygdaloid lesions. Although the effects of hippocampal lesions on water consumption immediately postoperatively have not been examined, Kimble \& Coover (1966) observed a $16 \%$ increase in water intake 20-37 days following hippocampal lesions. The sum (118\%) of these percentage increases is remarkably similar to the magnitude of the increase $(128 \%)$ observed in the present study during the first postoperative week. The same analysis can be applied to the data obtained during the fourth postoperative week. Harvey \& Hunt (1965) reported a $37 \%$ increase in water consumption four weeks (48-64\% six months postoperatively) following septal lesions. Grossman \& Grossman (1963) observed a $16 \%$ increase during the second two weeks after amygdaloid lesions. Hippocampal rats drank $16 \%$ more water than controls 20-37 days postoperatively (Kimble \& Coover, 1966). The sum (69\%) of these reported increases approximates the percentage increase (83\%) observed in this study during the fourth postoperative week.

Since the lesioned Ss evidenced a sustained increase in water consumption but only a transient increase in food intake, the data suggest that nippocampal, septal and amygdaloid regulation of food intake is mediated by pathways which differ from those concerned with regulation of water consumption. One possibility is that these structures influence food intake via their medial forebrain bundle connections with lateral preoptic and hypothalamic areas. The increase in food intake observed during the second postoperative week may reflect an attempt by the lesioned Ss to regain weight or to correct a possible initial electrolyte imbalance resulting from polyuria.

Additional work is necessary to determine whether the polydipsia observed is primary, that is, due to a direct effect on thirst, or secondary to a polyuria produced by suppression of antidiuretic hormone (ADH) release. The latter possibility is suggested by studies showing that stimulation of limbic structures can cause ADH secretion (Yoshida et al, 1966).

\section{References}

Grossman, S. P., \& Grossman, L. Food and water intake following lesions or electrical stimulation of the amygdala. Amer. $J$. Physiol., 1963, 205, 761-765.

Harvey, J. A., \& Hunt, H. F. Effect of septal lesions on thirst in the rat as indicated by water consumption and operant responding for water reward. J. comp. physiol. Psychol., 1965, 59, 49-56.

Kimble, D. P., \& Coover, G. D. Effects of hippocampal lesions on food and water consumption in rats. Psychon. Sci., 1966, 4, 91-92.

Konig, J. F. R., \& Klippel, R. A. The rat brain: A stereotaxic atlas of the forebrain and lower parts of the brain stem. Baltimore: Williams and Wilkins, 1963.

Lubar, J. F., \& Wolfe, J. W. Increased basal water and food ingestion in cingulectomized rats. Psychon. Sci., 1964, 1, 289-290.

Wolfe, J. W., Lubar, J. F., \& Ison, J. R. Effects of medial cortical lesions on appetitive instrumental conditioning. Physiol. \& Behav., 1966, in press.

Yoshida, S., Ibayashi, H., Murakawa, S., \& Nakao, K. Cerebral control of antidiuretic hormone release: Effect of electrical stimulation of the medial aspect of the dog brain. Endocrinology, 1966 , $79,871-874$.

\section{Noie}

1. This study was supported in part by USPHS grant MH-12844-01, S. A. Lorens, principal investigator. 